\title{
Des profils et de la profondeur
}

\section{Dave Lüthi}

\section{OpenEdition}

Journals

Édition électronique

URL : http://journals.openedition.org/edl/957

DOI : $10.4000 /$ edl. 957

ISSN : 2296-5084

\section{Éditeur}

Université de Lausanne

\section{Édition imprimée}

Date de publication : 15 mars 2017

Pagination : 7-12

ISBN : 978-2-940331-64-2

ISSN : 0014-2026

\section{Référence électronique}

Dave Lüthi, « Des profils et de la profondeur », Études de lettres [En ligne], 1 | 2017, mis en ligne le 15 mars 2019, consulté le 19 décembre 2020. URL : http://journals.openedition.org/edl/957 ; DOI : https://doi.org/10.4000/edl.957 


\section{DES PROFILS ET DE LA PROFONDEUR}

Ce troisième volume d'Études de Lettres consacré à l'étude de l'architecte comme "professionnel de l'art de construire" s'intéresse à définir son profil - ou ses profils, tout dépendant de l'angle de vue envisagé. Comme un observateur le ferait d'une sculpture juchée sur un piédestal, les auteur.e.s réuni.e.s dans ce volume tournent autour de l'architecte, érigé en objet d'étude, le regardant avec des interrogations variées portant sur sa formation, son entourage social, sa liberté créatrice et les limites qu'il s'impose à lui-même par le biais des règlements qu'il a contribué à mettre sur pied pour réguler sa propre pratique. Tourner autour, c'est définir, délimiter, regarder à distance ou observer le détail, envisager l'objet dans son épaisseur, sa réalité, sa tridimensionnalité, ce qui implique qu'une partie reste toujours dans l'ombre ou, du moins, invisible, cachée. Mais il faut l'accepter: en s'intéressant au profil, on ne s'intéresse pas tant à la masse qu'à la surface et aux interactions entre l'architecte et le monde qui l'entoure. Lecture superficielle, formelle? De loin s'en faut.

Plusieurs approches thématiques, souvent différentes d'un point de vue méthodologique, sont envisageables pour définir la profession d'architecte aux époques moderne et contemporaine. La monographie reste la plus courante, même si sa forme et sa fonction suscitent toujours questions et débats: décrire "sa vie, son ouvre» demeure un exercice périlleux en raison des sources à disposition et de la nature même de l'acte créatif, souvent partagé entre plusieurs acteurs difficiles à repérer et à identifier avec précision. Nous avons dans cette même collection tenté une telle perspective en questionnant le statut professionnel de l'architecte et son évolution, du maître maçon polyvalent sans véritable définition professionnelle à l'architecte «artiste» ou à l'agence d'architectes au début du 
$\mathrm{XX}^{\mathrm{e}}$ siècle ${ }^{1}$. Une autre approche, très fructueuse, a aussi été envisagée: celle de l'étude de la clientèle de l'architecte, qui a montré qu'il n'est pas toujours simple de différencier le maître d'ouvrage du maître d'œuvre à l'époque contemporaine ${ }^{2}$. Le rapport entre l'architecte et son client est apparu comme l'un des pans des relations commerciales pratiquées par le constructeur, qui peut aussi bâtir à son compte (ou celui de sociétés immobilières) sans avoir besoin de se soucier des attentes d'une clientèle de locataire de plus en plus standardisée. On a pu aussi, enfin, conjuguer plusieurs mises en perspective en travaillant à l'écriture de monographies professionnelles, traitant des points précédemment évoqués en lien avec le corpus bâti du ou des praticiens envisagés ${ }^{3}$. Il faut relever ici à quel point les technologies numériques renouvellent notre approche et nos connaissances, sans pourtant invalider les méthodes plus traditionnelles ${ }^{4}$. Ce volume de Profils s'inscrit dans la continuité de cet essai «synthétique», si l'on peut dire, en faisant se côtoyer des thématiques a priori éloignées, mais qui circonscrivent toutes, en fait, l'architecte "professionnalisé» ou en cours de le devenir aux XIX et $\mathrm{XX}^{\mathrm{e}}$ siècles.

Les thématiques développées lors du colloque international organisé à l'Université de Lausanne les 25-26 septembre 2015, qui a réuni des conférencières et conférenciers de Suisse, de France et d'Allemagne, couvrent en effet des champs variés, mais essentiels à la compréhension des phénomènes et des habitus qui caractérisent le commun des constructeurs de l'époque contemporaine. Formation - ici évoquée à partir de l'exemple essentiel de l'École des beaux-arts de Paris, que 340 architectes suisses ont fréquentée -, associations professionnelles et autopromotion, contrôle réglementaire de la pratique, déplacement professionnel volontaire ou forcé (le cas de Strasbourg est emblématique à cet égard), acclimatation à une nouvelle ambiance architecturale, formation et déformation du bureau d'architecture sont autant de thèmes ici abordés pour retracer un certain quotidien des architectes; pas celui de la création, dont témoignent les esquisses, les plans et les bâtiments euxmêmes, mais celui de tous ces moments ne faisant pas partie directement

I. G. Cassina, D. Lüthi (dir.), La profession d'architecte en Suisse romande.

2. D. Lüthi (dir.), Le client de l'architecte.

3. D. Lüthi (dir.), Trajectoires d'architectes vaudois.

4. Nous renvoyons aussi au mémoire de maîtrise de G. Curchod, Charles-François Bonjour (1870-1961), qui a montré les apports de la conjugaison des archives avec ces technologies. 
du processus créatif et qui, pourtant, définissent autant que leurs œuvres les architectes comme des artistes, mais aussi des hommes d'affaires, voire parfois comme des commerçants...

En dépit d'une approche souvent microhistorique affirmée et assumée - le recours à des fonds d'archives peu exploités assurant le renouvellement des questionnements -, les objets développés ici acquièrent une valeur bien plus exemplaire par leur juxtaposition, notamment, mais aussi par l'éclairage mutuel qu'ils s'apportent les uns aux autres: l'étude de la formation expliquant les formes d'associations dans les bureaux, le phénomène sociétaire trouve écho dans la multiplication de règlements voulus souvent par de véritables lobbies, etc. Certes, ces études de cas ne sont pas généralisables: en revanche, elles soulèvent des questionnements qu'il faudra peu à peu transposer à d'autres cadres pour en valider la pertinence et susciter des études transversales.

Deux thématiques reliées à la géographie artistique, qui stimule depuis longtemps la recherche lausannoise en histoire de l'art, peuvent être mises en évidence. D’une part, le rapport de la périphérie au centre est diversement traité dans les articles de notre recueil. Le rapport de la Suisse romande à Paris, essentiel, est ausculté depuis Paris à travers la liste des centaines d'étudiants inscrits à l'École des beaux-arts; les effets de miroir entre Paris et sa province apparaissent avec force dans l'étude des nombreuses publications des sociétés d'architectes qui fleurissent dans les centres régionaux; enfin, la position très spécifique de Strasbourg, situé dans un Reichsland dépendant directement de l'empereur, est aussi interrogée. Centre régional, mais périphérie de l'Empire, la ville qui connaît un essor urbain phénoménal après son passage à l'Allemagne en 1871, assure de fait une certaine indépendance aux architectes. La comparaison de ces trois cas est tout à fait nouvelle et pertinente: il y a différents types de centres, et différentes sortes de périphéries. Elle donne en tous les cas à réfléchir sur la définition de la périphérie, qui est bien moins «en retard " et par conséquent "passive» - pour reprendre les termes développés dans un tout autre cadre par Enrico Castelnuovo et Carlo Ginzburg,

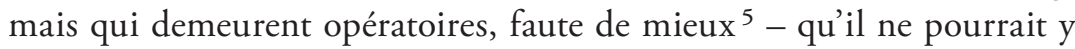
paraître de prime abord. Le cas helvétique montre que si les architectes se forment pour beaucoup à Paris, il n'y a pas d'aveuglement non plus face

5. E. Castelnuovo, C. Ginzburg, «Domination symbolique et géographie artistique», p. 61. 
à un modèle professionnel qui ne peut être importé tel quel: le pragmatisme, tant architectural que commercial, prime.

Plutôt que des périphéries, et c'est la deuxième thématique que nous aimerions signaler ici, Strasbourg et la Suisse romande apparaissent de plus en plus comme des zones frontières au statut très spécifique, «double périphérie», toujours selon Castelnuovo et Ginzburg, qui "posent la question du choix à travers l'assimilation, la résistance ou la combinaison ${ }^{6}$ et dont on perçoit peu à peu l'originalité face aux centres. Un architecte lausannois ou strasbourgeois a donc peut-être plus de liberté d'action dans un contexte professionnel où les codes en vigueur dans les centres perdent une partie de leur sens, ce qu'il faut en partie compenser par une adaptation aux habitudes locales ou par la mise en place de stratégies nouvelles de placement social et professionnel, qui individualisent fortement les parcours individuels ${ }^{7}$.

Cette approche comparative a le grand avantage de mettre en évidence des caractéristiques topiques ou régionales de la profession d'architecte. Ses contours sont très mobiles selon l'endroit où est établi le constructeur. À un moment donné, un architecte peut connaître plusieurs types de carrière selon son lieu d'établissement: se pose la question du choix du lieu; pourquoi un architecte suisse passé par Paris rentre-t-il au bercail, alors qu'il est sans doute conscient que sa carrière n'aura rien à voir avec celle de ses confrères français? Espère-t-il pouvoir faire évoluer la situation, se contente-t-il de ce qu'il connaît déjà, se fait-il une raison et si oui, quelle raison? Vaut-il mieux être architecte à Strasbourg qu'à Zurich ou qu'à Stuttgart? À Tours ou à Périgueux qu’à Paris?

Les profils ressemblent parfois au cheminement du regard sur une carte; les yeux s'arrêtent à un endroit précis, et le détail attire l'attention. C'est ainsi que cet ouvrage se présente: comme une succession de détails curieux qui définissent l'entier du portrait.

Dave LÜTHI

Université de Lausanne

6. F. Elsig, "Migrations artistiques», p. 9.

7. Voir à ce propos D. Lüthi (dir.), Trajectoires d'architectes vaudois. 


\section{BIBLIOGRAPHIE}

Cassina, Gaëtan, Lüthi, Dave (dir.), La profession d'architecte en Suisse romande (XVI $-X X^{e}$ siècle), Etudes de lettres 2009/1, Université de Lausanne, Faculté des Lettres, Lausanne, 2009.

Castelnuovo, Enrico, Ginzburg, Carlo, «Domination symbolique et géographie artistique", Actes de la recherche en sciences sociales, 40 (1981), p. 51-72.

Curchod, Guillaume, Charles-François Bonjour (1870-1961): un architecte régionaliste et son bureau lausannois à la Belle Epoque, Université de Lausanne, mémoire de maîtrise, 2016.

Elsig, Frédéric, "Migrations artistiques: quelques enjeux méthodologiques", Art + Architecture en Suisse, 58.3 (2007), p. 6-12.

Lüthi, Dave (dir.), Le client de l'architecte. Du notable à la société immobilière: les mutations du maître de l'ouvrage en Suisse au XIX siècle, Études de lettres 2010/4, Université de Lausanne, Faculté des Lettres, Lausanne, 2010.

- (dir.), Trajectoires d'architectes vaudois. Douze carrières de constructeurs des XIX et $X X^{e}$ siècles, numéro spécial de la Revue de généalogie vaudoise et d'histoire des familles, 2016. 
